\title{
Methodology for the Global Youth Tobacco Use Survey (GYST), Vietnam, 2014
}

\author{
Kim Bao Giang ${ }^{1}$, Hoang Van Minh' ${ }^{2}$ Phan Thi Hai ${ }^{3}$, Doan Thu Huyen ${ }^{3}$, Luong \\ Ngoc Khue ${ }^{3}$, Nguyen Thuy Linh ${ }^{3}$, Nguyen Tuan Lam ${ }^{4}$, Pham Thi Quynh Nga \\ Luu Ngoc Hoat ${ }^{1}$
}

\begin{abstract}
Viet Nam is a country with the highest rate of adult male smokers in the world. In 2010, the percentage of adult male smokers was 47.4\%. Each year in Viet Nam, there are 40,000 lives lost from tobacco-related diseases. The Global Tobacco Youth Survey is an international standardized survey that has been conducted in more than 140 countries. To provide evidence for tobacco control, Viet Nam already conducted two rounds of GYTS in 2003 and 2007. The GYTS in 2014 is the survey's third iteration. This paper aims to document key methodological details and socio-demographic characteristics of the respondents included in the 2014 GYTS in Viet Nam. This survey followed international protocol and was conducted in 13 provinces representative of 6 geographical regions. A total of 3,430 school children, aged 13 to 15 years used a standardized answer sheet to answer 76 questions about seven tobacco-related topics, including prevalence of tobacco use, environmental tobacco smoke, access and availability, media and advertisement, cessation, knowledge and attitudes, tobaccorelated school curriculum. This GYTS provides valid and reliable data for monitoring tobacco use among youth in Vietnam and is recommended to be regularly repeated.
\end{abstract}

Keywords: Tobacco-use - Global-Youth-Tobacco-Use-Survey (GYTS) - Viet-Nam

Asian Pac J Cancer Prev, 17 Tobacco Prevention and Control in Vietnam Suppl, 11-15

\section{Introduction}

Viet Nam is one of 15 leading countries with the highest rates of adult male smokers in the world. In the ASEAN region, Viet Nam is the third leading country with the highest rate of adult smokers. According to the Global Adult Tobacco Survey (GATS) in 2010 (Ministry of Health of Vietnam et al., 2010), the percentage of adult male smokers was $47.4 \%$. Each year in Viet Nam, there are 40,000 lives lost from tobacco-related diseases (Levy et al., 2006).

Exposure to second hand smoke has also been scientifically proven to cause deadly diseases (World Health Organization, 2003). The WHO recommends that no level of exposure to secondhand smoke is safe. In Viet $\mathrm{Nam}$, in parallel with the highest rate of active smoking in the world, the rate of secondhand smoking is also very high. The rate of non-smokers exposed to tobacco smoke is $73.2 \%$ at home and $55.9 \%$ at the workplace respectively; the rate of tobacco use in the south is found to be higher than the north. People of lower socioeconomic class tend to smoke more than those with higher incomes. In addition, higher income brackets tend to quit smoking at higher rates than those of lower income groups (Ministry of Health of Vietnam et al., 2010). Overall, tobacco consumption has a significant impact on the economy. In 2007, the Vietnamese population spent more than 14 trillion VND for tobacco products; this figure rose to 22,000 trillion VND in 2011. Among the poorest households in Viet Nam, the expenditure for tobacco use is 2.2 times higher than the expenditure for education and 1.6 times higher than that for health care services. The treatment cost for lung cancer, heart disease and COPD, only three out of 25 tobacco-related diseases, is up to 2,304 billion in 2010 (Ross et al., 2007).

Recognizing the importance of tobacco control, Viet Nam issued the National Policy on Tobacco Control in August 2000 (The Government of Vietnam, 2000) and Ratified the FCTC in December 2004 (The Government of Vietnam, 2009). In addition, Viet Nam has just achieved a very significant milestone in the approval of the country's first Tobacco Control Law by the National 
Assembly, consistent with the majority of the FCTC Articles in June 2012 . This law has been in effect since May 2013 (Vietnam National Assembly, 2012) with a number of important measures implemented with the objective of reducing the demand of both tobacco supply and consumption.

Aiming to help countries effectively monitor the smoking rate among youth, the World Health Organization (WHO), Tobacco Free Initiative, and the Center for Disease Control and Prevention (CDC) Office on Smoking and Health have developed the Global Youth Tobacco Survey (GYTS) (Centers for Disease Control and Prevention, 2007) as part of the Global Tobacco Surveillance System (GTSS) to assist countries in establishing tobacco control surveillance and monitoring programs (Warren et al., 2009). In Vietnam, this survey has now been conducted three times. In 2001, the GYTS survey was carried out in five provinces (Ha Noi, Hai Phong, Tuyen Quang, Da Nang, Ho Chi Minh). The results showed that cigarette smoking prevalence among students aged 13-15 years was $9 \%$ among males and $1.5 \%$ among females; with overall smoking prevalence at $4.09 \%$ (VINACOSH, 2003). A second round of the GYTS survey was carried out in 2007 in a total of 9 provinces/ cities (namely Hanoi, Hai Phong, Tuyen Quang, Hoa Binh, Hue, Da Nang, Lam-Dong, Ho Chi Minh city, and Tien Giang). The overall smoking prevalence among students aged 13-15 years old decreased slightly to $3.3 \%$ compared to the 2003 survey, which is relatively low compared to other countries in the Western Pacific Region (WPR). The smoking rate in boys is fairly high compared to girls (5.9\% vs. $1.2 \%)$. The gender difference in smoking rates among students was to some extent similar to that of other WPR countries (VINACOSH, 2007). The GYTS findings are used for advocacy and evidence-based planning and intervention, with a focus on the development of Tobacco Control Law in Viet Nam (formulation of a smoking ban effective for youth under 18 and within 100m from school gates, and comprehensive prohibition of TAPs). The Tobacco Control Law has been in effect since May 1 st, 2013, creating the necessary momentum for curbing youth smoking in Viet Nam.

The present third GYTS survey in 2014 was designed to support the development and monitoring of a panoramic assessment of youth smoking in Viet Nam, building on the reports which have already been published for all participating countries (Warren et al., 2008), South East Asia (Sirichotiratana et al., 2008; Sinha et al., 2011; 2014), South Asia (Kabir et al., 2013; Rao et al., 2014), and the Arab Gulf countries (Moh'd Al-Mulla et al., 2008). The GYTS Viet Nam 2014 assessed students' attitudes, knowledge and behaviors related to tobacco use and its health impact, including: cessation, environmental tobacco smoke exposure, media and advertising, minors' access and school curriculum.

As one of a cluster of publications on tobacco control in the present supllement of the Asian Pacific Journal of Cancer Prevention, this paper aims to document key methodological details and socio-demographic characteristics of the respondents in the GYTS Viet Nam 2014.

\section{Materials and Methods}

The Global Youth Tobacco Survey (GYTS) is a standardized, international survey. GYTS has been conducted in 140 countries, covering 11 territories and across all six WHO regions since 1999. This survey had been designed by several international experts to provide standardized methodology for constructing sampling frames, selecting schools and classes, preparing questionnaires, conducting field procedures, and processing data (Warren et al., 2008). Similar to GYTS in other countries, the scope of this GYTS in Viet Nam was defined through consultation among the country research coordinator, WHO Tobacco Free Initiative regional advisors, and CDC technical advisors.

\section{Sampling and sample size}

Sample size. The sample size was estimated under the technical advice of WHO and CDC. The desired number of schools was 40, with an expected school response rate of $80 \%$, resulting in an actual school sample size of of 50 . With an expected student attendance rate of $80 \%$ and a desired 2,400 student questionnaires, a minimum of 3000 students needed to be sampled. The final sample size used for analysis was 3430 school children aged 13 to 15 years.

Sampling. GYTS is a national school-based survey of students aged 13-15 years that makes use of a proportion to population size (PPS) methodology for constructing the sample frame and selecting schools / classes. GYTS is conducted based on the core questionnaires provided by the CDC, with the flexibility for adding questions specific to the local situation. Viet Nam's GYTS is a school-based cross-sectional survey with secondary school students and high school students aged 13-15 (grades 8-10). A two-stage sample design is employed to produce a representative sample of school students aged 13-15. In Viet Nam there is no national school enrolment lists at a central administrative level to provide a comprehensive lists of schools in each province and a total registrar of students in each school. Consequently, the total number of school children age 13-15 from secondary school in each province was first collected. From this list, CDC selected

Table 1. Number of Schools, Classes and Students Completing the Survey in Each Selected Province

\begin{tabular}{llccc}
\hline No Province & $\begin{array}{c}\text { No. } \\
\text { classes }\end{array}$ & $\begin{array}{c}\text { Total } \\
\text { students }\end{array}$ & $\begin{array}{c}\text { No. completed } \\
\text { questionnaire }\end{array}$ \\
\hline 1 & An Giang & 3 & 210 & 199 \\
2 & Ben Tre & 2 & 143 & 138 \\
3 & Daknong & 2 & 103 & 93 \\
4 & Dong Nai & 2 & 132 & 123 \\
5 & Ha Noi & 10 & 728 & 690 \\
6 & Hai Duong & 4 & 301 & 292 \\
7 & Ho Chi Minh & 6 & 549 & 465 \\
8 & Nghe An & 6 & 488 & 457 \\
9 & NinhBinh & 3 & 178 & 160 \\
10 & PhuTho & 4 & 278 & 265 \\
11 & Quang Ngai & 3 & 228 & 209 \\
12 & Quang Tri & 3 & 183 & 170 \\
13 & Yen Bai & 3 & 218 & 169 \\
\hline
\end{tabular}


Table 2. Surveyed Questions and Main Contents

\begin{tabular}{|c|c|c|}
\hline Topics & Number of questions & Main contents \\
\hline 1. Background information & 8 & $\begin{array}{l}4 \text { core questions: Age, sex, grade of students, weekly spending money, } \\
4 \text { optional added questions: father and mother's occupation, education levels. }\end{array}$ \\
\hline 2. Prevalence of tobacco use & 19 & $\begin{array}{l}\text { Tobacco use, initial age of smoking, frequency of smoking, per day number of } \\
\text { cigarettes used, smoking place, use of other types of tobacco, tobacco dependence. }\end{array}$ \\
\hline 3. Environmental tobacco smoke. & 7 & Exposure to tobacco smoke at home, at different public places \\
\hline 4. Access and availability & 7 & $\begin{array}{l}\text { Sources of tobacco, tobacco brands used, places to buy cigarettes, refuse to sell } \\
\text { cigarettes for children, types of tobacco purchased, cigarette price. }\end{array}$ \\
\hline 5. Media and advertisement & $6+8$ & $\begin{array}{l}\text { - Exposure, frequency and impact of exposure to anti smoking message in different } \\
\text { media means, sport and social events and health warning label. } \\
\text { - Exposure to tobacco advertisement in media, point of sales, events, internet, } \\
\text { tobacco marketing staff and receiving tobacco promotion products. }\end{array}$ \\
\hline 6. Cessation & 6 & $\begin{array}{l}\text { Desire to quit smoking, attemp to quit, perceived ability to quit, quit duration, } \\
\text { reason to quit, advice to quit }\end{array}$ \\
\hline 7. Knowledge and attitudes & 13 & $\begin{array}{l}\text { Smoking parents, knowledge and attitude about tobacco smoking, notice of } \\
\text { smoking at the school by teachers or friends }\end{array}$ \\
\hline 8. Tobacco-related school curriculum & 2 & School policy on tobacco smoking for teachers and children \\
\hline
\end{tabular}

$20 \%$ of provinces representing 6 geographic areas (13 provinces among 63 provinces in total) by PPS.

All schools containing grades 8-10 were included in the sampling frame. The two-stage clustered sample design was used to produce a representative sample of students in grades 8 to 10 .

\section{Questionnaire}

An international version of original questionnaire developed by the CDC consisted of 43 core questions recommended for use in surveying in all countries. This included 14 questions on shisha, 14 questions on Bidi use, 17 questions on smokeless tobacco use, 7 questions on school curriculum and 62 optional questions. The international version of the original questionaire was translated into Vietnamese and was adapted to the local context when necessary (Global Youth Tobacco Survey Collaborative Group, July 2012). After its adoption, it was translated into English and sent to CDC for consolidation and verification of no mistranslations. Prior to data collection, this process was assessed for ease of understanding and local cultural applicability.

The final questionnaire consisted of 76 questions, including 8 background questions. Core questions focussed on seven topics (Table 2).

\section{Data collection}

Data collection was coordinated by the Hanoi Medical University, with medical students and senior data collectors from the academic institution. All data collectors were carefully trained by the research team; the research team also participated in the pilot survey and revision of the questionnaire, including field work planning. Field work supervision was performed by the Hanoi Medical University and Viet Nam Steering Committee on Smoking and Health (VINACOSH). Three data collection teams were established, each consisting of 3-4 data collectors and a field supervisor. Instruction for data collection by the CDC was translated into Vietnamese for use (Preparing for the Survey, Training Survey Administrators, Administering the Survey and Preparing Data for Processing).
All the procedures of data collection followed CDC's guidance and templates. Selected data collectors visited selected classes to distribute the standardised questionnaire and answer sheets. At each selected class, the data collector introduced the survey, explained the meaning of each question, and how to use the answer sheet. Participants were informed of the confidentiality of their information and their rights to refuse survey participation. Participation was entirely voluntary and participants were asked to answer each question independently without any discussion with friends/classmates.

\section{Data processing and weighting}

Data processing was done by the CDC.All initial data processing (scanning, cleaning, editing, and weighting) was conducted at the CDC. Data analysis was done for students in the age range of 13 to 15 years.

The CDC created a weight system, associated with each participant to reflect the likelihood of sampling each student and to reduce bias by compensating for differing patterns of non-response. The weight used for estimation is given by:

$$
\mathrm{W}=\mathrm{W} 1 * \mathrm{~W} 2 * \mathrm{f} 1 * \mathrm{f} 2 * \mathrm{f} 3 * \mathrm{f} 4
$$

$\mathrm{W} 1=$ the inverse of the probability of selecting the school $\mathrm{W} 2=$ the inverse of the probability of selecting the classroom within the school

$\mathrm{f} 1$ = a school-level non-response adjustment factor calculated by school size category (small, medium, large) $\mathrm{f} 2=$ a class adjustment factor calculated by school

$\mathrm{f} 3$ = a student-level non-response adjustment factor calculated by class

$\mathrm{f} 4=\mathrm{a}$ post stratification adjustment factor calculated by gender and grade

\section{Ethical clearance}

The survey was approved by the Ministry of Health, Ministry of Education and Training (MoET) and the Department of Education and Training and schools from the 13 participating provinces/cities. The purpose, contents and survey plan were reported to MoET, DoET and selected schools. The survey protocol was approved by the ethical committee of the Hanoi Medical University. 
Table 3. Age and Sex Distributions of Study Participants

\begin{tabular}{|c|c|c|c|}
\hline \multicolumn{2}{|c|}{ Indicator } & Unweighted frequency & \multirow{2}{*}{$\begin{array}{c}\text { Weighted percent } \\
27.8\end{array}$} \\
\hline Age & 13 years old & 1,107 & \\
\hline & 14 years old & 1,232 & 35.0 \\
\hline & 15 years old & 1,091 & 37.2 \\
\hline \multirow{3}{*}{ Sex } & Male & 1,645 & 49.1 \\
\hline & Female & 1,781 & 50.9 \\
\hline & Missing & 4 & - \\
\hline
\end{tabular}

Table 4. Occupations of Study Participant Parents

\begin{tabular}{|c|c|c|c|c|}
\hline \multirow[t]{2}{*}{ Job } & \multirow{2}{*}{\multicolumn{2}{|c|}{$\begin{array}{c}\text { Father } \\
\text { Unweighted Weighted \% } \\
\text { frequency and } 95 \% \text { CI }\end{array}$}} & \multicolumn{2}{|c|}{ Mother } \\
\hline & & & $\begin{array}{l}\text { Unweighted } \\
\text { frequency }\end{array}$ & $\begin{array}{l}\text { Weight } \\
\text { and } 95\end{array}$ \\
\hline \multicolumn{5}{|c|}{ Farmer, fishing, forestry } \\
\hline & 1,483 & $37.8(36.1$ & 1,353 & $33.6(32$ \\
\hline \multicolumn{5}{|c|}{ Official and state employees } \\
\hline & 239 & $8.1 \quad(7.0-9.2)$ & 303 & $10.1)$ \\
\hline \multicolumn{5}{|c|}{ Army, police } \\
\hline & 100 & $3.0 \quad(2.4$ & 20 & 0.6 \\
\hline \multicolumn{5}{|c|}{ Manual workers/labourers } \\
\hline & 334 & $10.2 \quad(9.1-1$ & 297 & $9.1 \quad(8$. \\
\hline \multicolumn{5}{|c|}{ Businessman or tradesman } \\
\hline & 441 & $14.7 \quad(13.3-16)$ & 551 & $17.3(15$ \\
\hline \multicolumn{5}{|c|}{ Free labor, housework, handicraftsman } \\
\hline & 401 & $11.6(10.5-12.8)$ & 693 & $22.4(20$ \\
\hline \multicolumn{5}{|c|}{ Other occupations } \\
\hline & 339 & $11.6(10$. & 164 & 5.4 \\
\hline \multicolumn{5}{|c|}{ Unknown occupation } \\
\hline & 85 & $(2.2-3.5)$ & 42 & $(0.9-1.7)$ \\
\hline & wer 8 & & 7 & \\
\hline
\end{tabular}

All students were given an explanation about the purposes of the survey and their rights to refuse survey participation without any consequences.

\section{Results}

\section{Overall response rate}

The response rate of the schools was $100 \%$ as 51 of the 51 sampled schools participated. The response rate of selected classes was $100.0 \%$ as 110 of the 110 sampled classes participated. The students response rate was $95.0 \%$ as 3,553 of the 3,740 sampled students participated. Thus, the overall response rate was: $100.0 \% * 100.0 \% * 95.0 \%=$ $95.0 \%$. Among the 3,553 students completing the survey, only 3,430 were in the age range of 13 to 15 .

\section{General study participant information}

The distribution of the 3430 surveyed participants are stratified according to age and sex in Table 3 . The sample was quite balanced with regard to sex $(49.1 \%$ male children and $50.9 \%$ female children). The number of participants aged 15 years old was the highest (37.2\% vs. $35 \%$ and $27.8 \%$ ).

The most common parental occupation in this age demographic was agricultural farmer, followed by fishing and forestry (37.8\% fathers and $33.6 \%$ of mothers); followed by free labor, houseworker or handicraftsman (11.6\% of fathers and $22.4 \%$ of mothers) (Table 4 ).

There were $2.1 \%$ of fathers and $3 \%$ of mothers who did not receive any formal education. Most parents completed
Table 5. Education Levels of Study Participant Parents

\begin{tabular}{|c|c|c|c|}
\hline \multirow{2}{*}{\multicolumn{2}{|c|}{$\begin{array}{l}\text { Education Father } \\
\text { Unweighted Weighted \% } \\
\text { frequency and 95\% CI }\end{array}$}} & \multicolumn{2}{|c|}{ Mother } \\
\hline & & $\begin{array}{l}\text { Unweighted } \\
\text { frequency }\end{array}$ & $\begin{array}{l}\text { Weighted \% } \\
\text { and 95\% CI }\end{array}$ \\
\hline \multicolumn{4}{|c|}{ No attendance at school } \\
\hline 79 & $2.1(1.6-2.6)$ & 120 & $3(2.4-3.5)$ \\
\hline \multicolumn{4}{|c|}{ Haven't graduated primary yet } \\
\hline 352 & $9.6(8.6-10.7)$ & 337 & $9.4(8.4-10.4)$ \\
\hline \multicolumn{4}{|c|}{ Graduated primary school } \\
\hline 732 & $19.4(18.1-20.8)$ & 730 & $19.3(18-20.7)$ \\
\hline \multicolumn{4}{|c|}{ Graduated secondary school } \\
\hline 602 & $17.2(15.8-18.5)$ & 660 & $18.8(17$. \\
\hline \multicolumn{4}{|c|}{ Graduated high school } \\
\hline 552 & $16.3(15-17.7)$ & 511 & $15.6(14.2$ \\
\hline \multicolumn{4}{|c|}{ Graduated university/college/professional high school } \\
\hline 360 & $12.3(11-13.6)$ & 384 & $12.9(11.7-14.2)$ \\
\hline \multicolumn{4}{|l|}{ Postgraduate } \\
\hline 109 & 3.8( & 93 & $3.3(2.6-4.0)$ \\
\hline \multicolumn{4}{|l|}{ Don't know } \\
\hline 640 & $19.2(17.7-20.6)$ & 590 & $17.7(16.2-19.1)$ \\
\hline No answer 4 & - & 5 & \\
\hline
\end{tabular}

primary school or secondary school $(36.6 \%$ of fathers and $38.1 \%$ of mothers). There were $16.1 \%$ of fathers and $16.2 \%$ of mothers surveyed who had completed university education or higher (Table 5).

\section{Discussion}

The Global Youth Tobacco Survey is a national representative survey using an international, standardized protocol and questionnaire, with the participation of several national and international experts from $\mathrm{WHO}$ and CDC (Warren et al., 2008). The use of a standardized protocol together with the technical inputs from experts are important factors to ensure this survey's validity and reliability.

The availability of data from three iterations of surveying for core indicators and country-specific indicators allows for comparison of changes as an indicator of the effectiveness of current tobacco control amongst youths in Viet Nam.

Taking advantage of experience from two previous surveys, this survey was very well-organized with regards to data collector training, field planning and implementation. The use of simple multiple choice questions as well as a scanned answer sheet should be stated as another advantage of this survey as it helped to reduce misunderstanding and eliminate administrative errors common to the data entering process.

This survey, however, had some limitations. First, the quality of information in a self-administered and selfreported survey is highly dependent on the attitudes and perspectives of surveyed participants. At the same time, survey participants represented many different ages and socioeconomic groups, likely resulting in a diversity of perspectives, even for participants of the same age and grade.

As acknowledged in a previous paper, one limitation of this survey is that it only focuses on children aged 13 to 15 years who attend school, which is clearly not 
representative of all children aged 13 to 15 in Viet Nam. Furthermore, since data collection in each class and school occurred on only one day, data only represents individuals in attendance at school on the day of data collection (Warren et al., 2008). In addition, it is important to note that, use of different recall times in different questions may challenge children to remember facts and events. Finally, the modest sample size of GYTS 2014 in Viet Nam would limit stratified analysis for different socioeconomic and demographic sub-groups.

In conclusion, GYTS in Viet Nam 2014 is a national, representative survey among youths providing valid and reliable evidence for monitoring tobacco control among youths in Viet Nam. Although limitations exist, repeated surveys using this international standardized protocol is very important to monitor and provide evidence for tobacco control in Viet Nam.

\section{Acknowledgements}

We thank the VINACOSH (Viet Nam Steering Committee on Smoking and Health) and GYTS team of Viet Nam for making this data available. We also thank WHO's Tobacco Free Initiative (TFI) and CDC's Office on Smoking and Health for providing technical assistance. We acknowledge the financial support and coordination of the Hanoi School of Public Health, World Health Organization and the Tobacco Control Research group of Viet Nam Tobacco Control Fund. We would lile to thank Viet Nguyen for performing English editing. We also would like to express our sincere appreciation to Dr. Malcolm Moore, the Editor-in-Chief of the Asian Pacific Journal of Cancer Prevention, for his significant input for this paper.

\section{References}

Global Youth Tobacco Survey Collaborative Group (2012). Global Youth Tobacco Survey (GYTS) : Core Questionnaire with Optional Questions.

Kabir MA, Goh KL, Khan MH (2013). A cross-country comparison of tobacco consumption among youths from selected South-Asian countries. BMC Public Health, 13, 379.

Levy DT, Bales S, Lam NT, et al (2006). The role of public policies in reducing smoking and deaths caused by smoking in Vietnam: Results from the Vietnam tobacco policy simulation model. Soc Sci Med, 62, 1819-30.

Ministry of Health of Vietnam, Hanoi Medical University, General Statistics Office of Vietnam (2010). Global Adult Tobacco Survey: Vietnam 2010.

Moh'd Al-Mulla A, Abdou Helmy S, Al-Lawati J, et al (2008). Prevalence of tobacco use among students aged 13-15 years in Health Ministers' Council/Gulf Cooperation Council Member States, 2001-2004. J Sch Health, 78, 337-43.

Rao S, Aslam SK, Zaheer S, Shafique K (2014). Anti-smoking initiatives and current smoking among 19,643 adolescents in South Asia: findings from the Global Youth Tobacco Survey. Harm Reduct J, 11, 8.

Ross H, Trung DV, Phu VX (2007). The costs of smoking in Vietnam: the case of inpatient care. Tobacco Control, 16, 405-9.

Sinha DN, Palipudi KM, Jones CK, et al (2014). Levels and trends of smokeless tobacco use among youth in countries of the World Health Organization South-East Asia Region. Indian J Cancer, 51 Suppl 1, S50-3.

Sinha DN, Palipudi KM, Rolle I, et al (2011). Tobacco use among youth and adults in member countries of South-East Asia region: review of findings from surveys under the Global Tobacco Surveillance System. Indian J Public Health, 55, 169-76.

Sirichotiratana N, Sovann S, Aditama TY, et al (2008). Linking data to tobacco control program action among students aged 13-15 in Association of Southeast Asian Nations (ASEAN) member states, 2000-2006. Tob Control, 17, 372-8.

Vietnam National Assembly (2012). Law on Tobacco Control. VINACOSH (2003). The 2003 GYTS in Vietnam: A preliminary report on youth tobacco use. Hanoi, Ministry of Health.

VINACOSH (2007). The 2007 GYTS in Vietnam: A preliminary report on youth tobacco use. Hanoi, Ministry of Health.

Warren CW, Jones NR, Peruga A, et al (2008). Global Youth Tobacco Surveillance, 2000--2007. MMWR, 57, 1-21.

Warren CW, Lee J, Lea V, et al (2009). Evolution of the Global Tobacco Surveillance System (GTSS) 1998-2008. Glob Health Promot, 16 (2 Suppl), 4-37. 